TIFR/TH/93-64

December 1993

\title{
Signatures of an Invisibly Decaying Higgs Particle at LHC
}

\author{
Debajyoti Choudhury ${ }^{a}$ and D.P. Roy $b$ 它 \\ a Max-Planck-Institut fur Physik, D-80805 Munchen, Germany \\ ${ }^{b}$ Theory Group, Tata Institute of Fundamental Research, Bombay 400005 , \\ India
}

\begin{abstract}
The Higgs particle can decay dominantly into an invisible channel in the Majoron models. We have explored the prospect of detecting such a Higgs particle at LHC via its associated production with a gluon, $\mathrm{Z}$ or $\mathrm{W}$ boson. While the signal/background ratio is too small for the first process, the latter two provide viable signatures for detecting such a Higgs particle.
\end{abstract}

\footnotetext{
${ }^{1}$ E-mail : DPROY@TIFRVAX.BITNET
} 
The signatures for Higgs particle detection at LEP and the future hadron colliders (LHC/SSC) have been extensively studied in the framework of the standard model (SM) and its supersymmetric (SUSY) extensions [1,2]. There exist some extensions of the SM, however, with a qualitatively different signature for the Higgs particle. These extensions are generically called the Majoron models [3-7] and have been quite popular e.g. in the context of generating neutrino mass. They are characterized by the existence of a Goldstone boson (the Majoron). Since the coupling of this Goldstone boson to the Higgs particle is not required to be small on any theoretical or phenomenological grounds, the Higgs particle could decay into an invisible channel containing a Majoron pair [7-9]. Indeed the importance of extending the Higgs search to this invisible decay channel has been repeatedly emphasized over the past decade $[8,9]$. However, quantitative investigations along this line have only started very recently $[10,11]$.

The key features shared by essentially all Majoron models is a spontaneously broken global $U(1)$ symmetry and a complex $S U(2) \times U(1)$ singlet scalar field $\eta$ transforming nontrivially under the global $U(1)$. The spontaneous breaking of the global $U(1)$ generates a massless Goldstone boson, the Majoron $J \equiv \operatorname{Im} \eta / \sqrt{2}$, and a massive scalar $\eta_{R} \equiv \operatorname{Re\eta } / \sqrt{2}$. The latter mixes with the massive neutral component $\phi_{R}$ of the standard Higgs doublet through a quartic term $\phi^{\dagger} \phi \eta^{\dagger} \eta$ in the scalar potential. Thus one has two massive physical scalars

$$
H=\cos \theta \phi_{R}+\sin \theta \eta_{R}, \quad S=\cos \theta \eta_{R}-\sin \theta \phi_{R},
$$

where the mixing angle can be chosen to lie in the range $0-45^{\circ}$, so that the $H$ and $S$ have dominant doublet and singlet components respectively. The above quartic term also generates the following couplings of $H$ and $S$ to the massless Goldstone boson $J$ :

$$
\mathcal{L}=\frac{\left(\sqrt{2} G_{F}\right)^{1 / 2}}{2} \tan \beta\left[M_{S}^{2} \cos \theta S J^{2}-M_{H}^{2} \sin \theta H J^{2}\right],
$$

where $\tan \beta=\langle\phi\rangle /\langle\eta\rangle$ is the ratio of the two vacuum expectation values $[8,9]$. The resulting decay widths of $H, S$ into the invisible channel $(J J)$ relative to the dominant SM channel $(b \bar{b})$ are

$$
\Gamma_{H \rightarrow J J} / \Gamma_{H \rightarrow b \bar{b}} \simeq \frac{1}{12}\left(\frac{M_{H}}{m_{b}}\right)^{2} \tan ^{2} \theta \tan ^{2} \beta\left(1-\frac{4 m_{b}^{2}}{M_{H}^{2}}\right)^{-3 / 2},
$$




$$
\Gamma_{S \rightarrow J J} / \Gamma_{S \rightarrow b \bar{b}} \simeq \frac{1}{12}\left(\frac{M_{S}}{m_{b}}\right)^{2} \cot ^{2} \theta \tan ^{2} \beta\left(1-\frac{4 m_{b}^{2}}{M_{S}^{2}}\right)^{-3 / 2} .
$$

The large mass ratio $\left(M_{H, S} / m_{b}\right)^{2}$ on the rhs implies that the invisible decay channel could dominate for $S$ as well as $H$ over a large range of the parameters $\tan \theta$ and $\tan \beta$. ${ }^{2}$

Although the eqs. (1-4) above were derived for the simplest model [3] having 1 singlet and 1 doublet scalar fields, similar considerations hold for those having a larger Higgs content [5-8] or a larger global symmetry group than $U 1[12]$. It may be added here that, the Higgs particles can also decay invisibly in the SUSY models via a pair of lightest superparticles (LSP). For the minimal supersymmetric standard model (MSSM) this invisible decay mode has been shown to dominate only over a tiny range of parameters for the scalar Higgs particles but over a larger range for the pseudoscalar [13].

Thus it is important to extend the Higgs search strategies at LEP and LHC to cover the possibility of a dominantly invisible decay. This is simple at LEP, since the dominant channel for Higgs search is the same for the SM and the invisible decays - i.e. the missing energy channel with one or two jets $[10,11]$. It corresponds to the Bjorken production process

$$
e^{+} e^{-\stackrel{Z}{\rightarrow}} Z^{*} H
$$

followed by $Z^{*} \rightarrow \nu \bar{\nu}, H \rightarrow b \bar{b}$ for the $\mathrm{SM}$ decay and $Z^{*} \rightarrow q \bar{q}, H \rightarrow$ $J J$ for the invisible decay. Indeed the larger branching fraction of $Z^{*}$ into quarks implies a larger event rate for the latter case. On the other hand the production cross-section would be suppressed by a factor of $\cos ^{2} \theta$ in this case. Combining the two effects leads to a $M_{H}$ bound in the Majoron models, which is within $\pm 6 \mathrm{GeV}$ of the $\mathrm{SM}$ value irrespective of the model parameters - i.e. $60 \pm 6 \mathrm{GeV}[10,11]$. A similar correlation between the Higgs signatures for the two models is expected to hold at LEP-II as well.

The present work is devoted to a systematic exploration of the signatures for an invisibly decaying Higgs particle search at the proposed large hadron collider (LHC). To start with one notes that the missing energy is not a measurable quantity at a hadron collider due to the large energy loss along the beam pipe. One has to consider instead the missing transverse momentum

\footnotetext{
${ }^{2}$ Both the parameters depend on the scale of the global $U(1)$ breaking relative to the $S U(2) \times U(1)$ breaking scale, on which there are no severe phenomenological constraints.
} 
$\left(p_{T}\right)$. Thus one must look at one of the following associated production processes which dominate Higgs production at large $p_{T}-(\mathrm{i}) \mathrm{H}+$ jet, (ii) $\mathrm{H}$ $+\mathrm{Z}$, (iii) $\mathrm{H}+\mathrm{W}$ and (iv) $\mathrm{H}+\mathrm{t} \overline{\mathrm{t}}$. We shall present below the results of our analysis for the first three processes. We shall see that the associated production of $\mathrm{H}$ with jet has too large a background to be a viable channel. On the other hand the associated production of $\mathrm{H}$ with $\mathrm{W}$ or $\mathrm{Z}$ bosons are expected to give viable signals. We shall not discuss the associated production of $\mathrm{H}$ with $\mathrm{t} \overline{\mathrm{t}}$, since it has been analysed recently in [15]. We shall only comment on the relative merit of this channel vis a vis $\mathrm{H}+\mathrm{W}(\mathrm{Z})$.

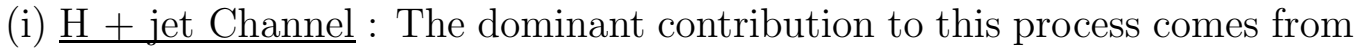
gluon-gluon fusion via the top quark loop

$$
g g \rightarrow g H,
$$

followed by the invisible decay $H \rightarrow J J$. The cross-section for (6) was computed for SSC/LHC energies in $[16]$ in the $m_{t} \rightarrow \infty$ limit and in $[17,18]$ for finite values of $m_{t}$. In the latter case the formalism is quite involved, containing several dilogarithmic functions. The cross-section presented below has been calculated for $m_{t}=160 \mathrm{GeV}$ using the code of ref. [18]. The dominant background comes from the tree-level process

$$
q g \rightarrow q Z
$$

followed by $Z \rightarrow \nu \bar{\nu}$. The contribution from $q \bar{q} \rightarrow g Z$ is about an order of magnitude smaller.

Figure 1 shows the jet $+\not p_{T}$ cross-section coming from the signal process (6) for $M_{H}=120 \mathrm{GeV}$ along with the background (7) at the LHC energy of 14 $\mathrm{TeV}$ and a rapidity cut of $\left|y_{j e t}\right|<5$. The two sets of curves shown have been calculated using the structure functions and the QCD coupling parameters of DFLM [19] and MRS [20]. Although the quantitative estimate of the crosssections seems to depend appreciably on this choice, the background is seen to be at least a factor of 40 larger than the signal. We have checked that reducing the rapidity cut leads to only a marginal decrease of this factor. Thus the $\mathrm{H}+$ jet channel does not provide a viable signature for an invisibly decaying Higgs particle.

\footnotetext{
${ }^{3}$ These two channels have been considered before in [14]. However, the results presented there have limited utility, since they do not contain the $\not_{T}$ distributions nor the effects of any $p_{T}$ cuts on the signals and the corresponding backgrounds.
} 
(ii) $\underline{\mathrm{H}+\mathrm{Z} \text { Channel }}$ : The dominant contribution to this signal comes from the associated Bjorken process

$$
q \bar{q} \stackrel{Z^{*}}{\rightarrow} H Z
$$

followed by $H \rightarrow J J$ and $Z \rightarrow \ell^{+} \ell^{-}$, where $\ell$ represents both electron and muon. The dominant background comes from

$$
q \bar{q} \rightarrow Z Z
$$

followed by $\nu \bar{\nu}$ decay of one $Z$ and $\ell^{+} \ell^{-}$decay of the other. There are also one-loop contributions to the signal from $g g \rightarrow Z H$ (via top quark) and the background from $g g \rightarrow Z Z$. The former is expected to add $\sim 10 \%$ to the signal [21] and the latter $\sim 30 \%$ to the background [22] at the LHC energy. Moreover the $p_{T}^{Z}$ dependence of the one-loop and the corresponding tree-level cross-sections are very similar, particularly for the background. Thus including these one-loop contributions would only reduce the signal/background ratio by $\sim 20 \%$. Therefore, it is adequate for our purpose to restrict to the dominant tree-level contributions.

We have calculated the dilepton $+\not p_{T}$ cross-sections coming from the signal (8) and the background (9) for the LHC energy of $=14 \mathrm{TeV}$ and the following acceptance cuts [23],

$$
\left|y_{p_{T}}\right|<5,\left|y_{\ell}\right|<3, p_{T}^{\ell}>20 \mathrm{GeV} .
$$

Figure 2 shows the $p_{T}$ distributions of the signal and background crosssections for $M_{H}=120$ and $160 \mathrm{GeV}$, assuming the DFLM structure functions [19]. We have checked that using the MRS [20] (EHLQ [24]) structure functions instead would raise (lower) these cross-sections by only $\sim 10 \%$. While the cross-sections are seen to go down rapidly with increasing $p_{T}$, there is a marked enhancement of the signal/background ratio. In particular, for a $p_{T}>200 \mathrm{GeV}$ cut, the signal becomes about half the size of the background. Of course the reason for this is that, while the signal (8) is a s-channel process, the background (9) has an additional suppression factor coming from the t-channel propagator.

Table 1 shows the integrated signal and background cross-sections for the missing- $p_{T}$ cuts of $p_{T}>100$ and $200 \mathrm{GeV}$. 7 Since the size of the $Z Z$ back-

\footnotetext{
${ }^{4}$ While the typical missing $-p_{T}$ cut assumed for LHC studies is $p_{T}>200 \mathrm{GeV}$ [23], we feel that the accompanying dilepton pair should make it feasible to reduce this cut to 100 $\mathrm{GeV}$.
} 
ground can be estimated from the $\ell^{+} \ell^{-} \ell^{+} \ell^{-}$channel, it should be possible to extract the $Z H$ signal if

$$
S / \sqrt{B} \gtrsim 5
$$

where $S$ and $B$ denote the numbers of signal and background events. Table 1 lists this ratio for both the low and high luminosity options of LHC, corresponding to typical integrated luminosities of 10 and 100 events/fb respectively. One sees from this list that it should be possible to search for an invisibly decaying Higgs particle upto $M_{H}=120 \mathrm{GeV}(200 \mathrm{GeV})$ at the low (high) luminosity option of LHC. This assumes of course a modest mixing angle $\left(\cos ^{2} \theta \simeq 1\right)$, so that one does not pay an appreciable price at the production vertex.

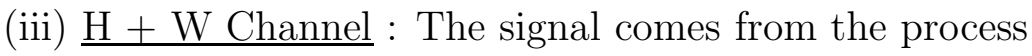

$$
q \bar{q}^{\prime} \stackrel{W^{*}}{\rightarrow} W H
$$

followed by the decays $W \rightarrow \ell \nu$ and $H \rightarrow J J$, resulting in lepton $+\not p_{T}$ events. The corresponding irreducible background is from

$$
q \bar{q}^{\prime} \rightarrow W Z
$$

followed by $W \rightarrow \ell \nu$ and $Z \rightarrow \nu \nu$. There is additional background from $W+$ multijet events, which can be effectively suppressed however by demanding a transverse mass cut

$$
M_{\ell p_{T}}=\left[\left(p_{T}^{\ell}+\not p_{T}\right)^{2}-\left(\vec{p}_{T}^{\ell}+\vec{p}_{T}\right)^{2}\right]^{1 / 2}>100 G e V
$$

This constraint is automatically satisfied by the above signal (as well as the irreducible background) process for $\not_{T}>200 \mathrm{GeV}$. Therefore, we shall concentrate on this region of missing- $p_{T}$. There remains one potentially serious background here - i.e. from

$$
t \bar{t} \rightarrow W W b \bar{b} \rightarrow \ell \nu \ell \nu b \bar{b}
$$

where $p_{T}$ of one of the leptons (including $\tau$ ) is $<20 \mathrm{GeV}$, so that it can not be identified. The size of this background is an order of magnitude larger than the signal (12). Note, however, that the signal (12) has no hadronic jet activity apart from those coming from the initial state QCD radiation, 
which would be soft and/or collinear with the beam. Therefore, it should be possible to suppress the above background (15) by a suitable $p_{T}$ and/or angular cut on the accompanying hadronic $(b)$ jets. But we have not been able to pursue this quantitatively since we had no access to an initial state QCD radiation program.

The lepton $+p_{T}$ cross-sections coming from the signal (12) and the irreducible background (13) have been calculated for the LHC energy of $14 \mathrm{TeV}$ and the cuts of eq. (10). These cross-sections are again insensitive to the choice of structure functions. Figure 3 shows the $p_{T}$ distributions of the signal and background for $M_{H}=120$ and $160 \mathrm{GeV}$ using the DFLM structure functions. There is again a marked improvement in the signal/background ratio with increasing $\not_{T}$. Indeed in the region of our interest, $\not_{T}>200 \mathrm{GeV}$, the signal is as large as the background.

Table 1 shows the integrated cross-sections for the signal and background for $\not_{T}$ cuts of $>100$ and $200 \mathrm{GeV}$. But we shall concentrate on the 200 $\mathrm{GeV}$ cut for this process for the reason mentioned above. In this case the $S / \sqrt{B}$ seems to be viable for the invisibly decaying Higgs particle search upto $M_{H}=200 \mathrm{GeV}$ even at the low luminosity option of LHC. And at the high luminosity option one can even afford to make generous allowance for a suppression factor $\left(\cos ^{2} \theta\right)$ at the production vertex. P One should of course bear in mind the above mentioned reducible background from (15).

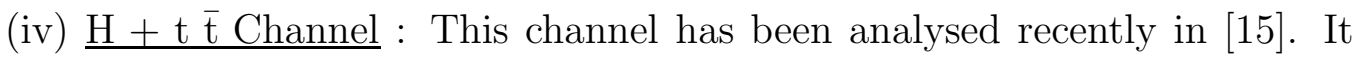
is useful to compare the resulting signal with those of the $Z H$ and $W H$ channels. The final state consists of $\ell, p_{T}$ and 4 jets from the leptonic decay of one of the $t$ quarks and hadronic decay of the other while $H$ decays invisibly. With a $p_{T}>200 \mathrm{GeV}$ cut, the signal is comparable to the background for $M_{H}=140 \mathrm{GeV}$; but the signal size is only $\sim 0.5 \mathrm{fb}$. Thus it can only be viable at the high luminosity option of LHC [15]. Besides this signal is far more demanding on detector performance since it requires $b$ identification as well as reconstruction of $W$ and $t$ masses from hadronic jets. However, as emphasized in [15], this is the only channel available for detecting an invisibly decaying pseudoscalar Higgs boson of the SUSY model, since it does not couple to $Z Z$ and $W W$ channels.

\footnotetext{
${ }^{5}$ It may not be necessary to extend the probe beyond $M_{H}=200 \mathrm{GeV}$, since in this case the $H \rightarrow W W, Z Z$ modes are expected to dominate over the $H \rightarrow J J$ (or a pair of LSP).
} 
In summary, the Higgs boson can have dominantly invisible decay in the Majoron models as well as some SUSY models. We have explored the prospects of detecting such a Higgs particle at LHC in the $\mathrm{H}+$ jet, $\mathrm{H}+\mathrm{Z}$ and $\mathrm{H}+\mathrm{W}$ channels. While the signal is expected to be overwhelmed by the background in the first case, one expects viable signals in the $\mathrm{H}+\mathrm{Z}$ and $\mathrm{H}+\mathrm{W}$ channels.

We are grateful to Nigel Glover for providing us with the code for computing the $g H$ cross-section as well as his collaboration in some of these computations. Indeed it is only by his insistence that his name appears here instead of the first page of this paper. We are also grateful to Rohini Godbole, Anjan Joshipura, Saurav Rindani and James Stirling for a number of useful comments. 


\section{$\underline{\text { References }}$}

1. See e.g. J.F. Gunion, H. Haber, G.L. Kane and S. Dawson, The Higgs Hunter's Guide (Addison-Wesley, Reading, MA, 1990).

2. ALEPH Collaboration: D. Decamp et al, Phys. Rep. 216 (1992) 253.

3. Y. Chikashige, R.N. Mohapatra and R.D. Peccei, Phys. Lett. 98B (1980) 265.

4. D.B. Reiss, Phys. Lett. 115B (1982) 217; F. Wilczek, Phys. Rev. Lett. 49 (1982) 1549.

5. G. Gelmini and M. Roncadelli, Phys. Lett. B99 (1981) 411.

6. A.S. Joshipura and S.D. Rindani, Phys. Rev. D46 (1992) 3000;

A.S. Joshipura and J.W.F. Valle, Nucl. Phys. B397 (1993) 105.

7. RindaniJ.C. Ramao, F. de Campos and J.W.F. Valle, Phys. Lett. B292 (1992) 329.

8. R.E. Schrock and M. Suzuki, Phys. Lett. B110 (1982) 250;

L.F. Li, Y. Liu and L. Wolfenstein, Phys. Lett. B159 (1985) 45;

E.D. Carlson and L.B. Hall, Phys. Rev. D40 (1985) 3187;

G. Jungman and M.A. Luty, Nucl. Phys. B361 (1991) 24.

9. A.S. Joshipura and S.D. Rindani, Phys. Rev. Lett. 69 (1992) 3269.

10. B. Brahmachari, A.S. Joshipura, S.D., D.P. Roy and K. Sridhar, Phys. Rev. D48 (1993) 4224.

11. ALEPH Collaboration: D. Buskulic et al, Phys. Lett. B313 (1993) 312.

12. J.D. Bjorken, Invited Talk at the Symp. on SSC Laboratory, Corpus Christi, Texas, October 1991, SLAC-PUB-5673(1991).

13. A. Djouadi, J. Kalinowski and P.M. Zerwas, Z. Phys. C57 (1993) 569. 
14. S.G. Frederiksen, N.P. Johnson, G.L. Kane and J.H. Reid, SSCLpreprint-577, July 1992 (Unpublished). See also J.C. Romao, J.L. Diaz-Cruz, F. Campos and J.W.F. Valle, FTUV/92-39, Nov. 1992 (Unpublished).

15. J.F. Gunion, UCD-93-28, Revised version, December 1993.

16. M. Chaichian, I. Liede, J. Lindfors and D.P. Roy, Phys. Lett. B198 (1987) 416 and B205 (1988) 595E.

17. R.K. Ellis, I. Hinchliffe, M. Soldate and J.J. Van der Bij, Nucl. Phys. B297 (1988) 221.

18. U. Baur and E.W.N. Glover, Nucl. Phys. B339 (1990) 38.

19. M. Diemoz, F. Ferroni, E. Longo and G. Martinelli, Z. Phys. C39 (1988) 21. We have used a parametrisation of these structure functions by M.G. Gluck, R.M. Godbole and E. Reya, DO-TH-89/16.

20. A.D. Martin, R.G. Roberts and W.J. Sterling, Phys. Rev. D47 (1993) 867.

21. B.A. Kniehl, Phys. Rev. D42 (1990) 2253.

22. E.W.N. Glover and J.J. Van der Bij, Nucl. Phys. B321 (1989) 561.

23. C. Albajar et al, Proc. of ECFA-LHC Workshop, Vol. II, CERN9010(1990) 621.

24. E. Eichten, I. Hinchliffe, K. Lane and C. Quigg, Rev. Mod. Phys. 56 (1984) 579. 
Table 1

The integrated cross-sections for the $Z H$ and $W H$ signals for $M_{H}=120,160,200 \mathrm{GeV}$ along with the corresponding backgrounds.

The signal $/ \sqrt{\text { background }}$ ratios are also shown for the integrated luminosity of 10 (100) events/ $f b$.

\begin{tabular}{|l|l|l||l|l|}
\hline Process & \multicolumn{2}{|c||}{$p_{T}>100 \mathrm{GeV}$} & \multicolumn{2}{c|}{$p_{T}>200 \mathrm{GeV}$} \\
\cline { 2 - 5 }$Z Z$ & $\sigma(f b)$ & $S / \sqrt{B}$ & $\sigma(f b)$ & $S / \sqrt{B}$ \\
\hline$Z H(120 \mathrm{GeV})$ & 9.0 & $5.9(18.6)$ & 1.9 & $3.2(10.2)$ \\
$Z H(160 \mathrm{GeV})$ & 5.3 & $3.5(10.9)$ & 1.4 & $2.4(7.5)$ \\
$Z H(200 \mathrm{GeV})$ & 3.3 & $2.2(6.8)$ & 1.1 & $1.9(5.9)$ \\
\hline$W Z$ & 38.5 & & 3.5 & \\
$W H(120 \mathrm{GeV})$ & 26.0 & & 3.3 & $5.9(18.6)$ \\
$W H(160 \mathrm{GeV})$ & 16.1 & & 2.7 & $4.8(15.2)$ \\
$W H(200 \mathrm{GeV})$ & 11.3 & & 2.4 & $4.3(13.5)$ \\
\hline
\end{tabular}




\section{Figure Captions}

Fig. 1. The Higgs signal and the $Z$ background cross-sections for the jet + missing- $p_{T}$ channel at LHC. The two sets of curves correspond to the DFLM [19] and MRS [20] structure functions.

Fig. 2. The $H Z$ signal (dotted and dashed lines) and the $Z Z$ background (solid line) cross-sections for the dilepton + missing- $p_{T}$ channel at LHC, calculated using the DFLM structure functions [19].

Fig. 3. The $H W$ signal (dotted and dashed lines) and the $W Z$ background (solid line) cross-sections for the lepton + missing- $p_{T}$ channel at LHC, calculated using the DFLM structure functions [19]. 
This figure "fig1-1.png" is available in "png" format from: http://arxiv.org/ps/hep-ph/9312347v1 
This figure "fig1-2.png" is available in "png" format from: http://arxiv.org/ps/hep-ph/9312347v1 
This figure "fig1-3.png" is available in "png" format from: http://arxiv.org/ps/hep-ph/9312347v1 